\title{
A Deductive-Nomological Model For MATHEMATICAL SCIENTIFIC EXPLANATION
}

\author{
EDUARDO CASTRO \\ Dep. Matemática, Universidade da Beira Interior \& LanCog, Centro de Filosofia, Universidade de Lisboa, PORTUGAL \\ ecastro@ubi.pt
}

\begin{abstract}
I propose a deductive-nomological model for mathematical scientific explanation. In this regard, I modify Hempel's deductive-nomological model and test it against some of the following recent paradigmatic examples of the mathematical explanation of empirical facts: the seven bridges of Königsberg, the North American synchronized cicadas, and HénonHeiles Hamiltonian systems. I argue that mathematical scientific explanations that invoke laws of nature are qualitative explanations, and ordinary scientific explanations that employ mathematics are quantitative explanations. I analyse the repercussions of this deductivenomological model on causal explanations.
\end{abstract}

Keywords: Explanation $\bullet$ mathematics $\bullet$ DN model $\bullet$ causation $\bullet$ science

\section{Introduction}

I cannot distribute exactly seven sardines evenly among my three cats without cutting any because there is a mathematical fact that states that seven cannot be divided evenly by three. This example seems to be a mathematical explanation of an empirical fact. That is, the mathematical fact that three is not a divisor of seven has an explanatory role in the content of the explanation.

There are two main views on the mathematical explanations of empirical facts. One view defends that mathematical explanations in science have the same nature as scientific explanations. For example, Lyon (2012) capitalises on the account of Jackson and Pettit (1990) for scientific explanations and argues that mathematical explanations are program explanations; on grounds of parsimony, Baker (2012, p.265) claims that mathematical explanations in science should be treated as scientific explanations. Moreover, Lange (2013) argues that mathematical explanations in science are non-causal explanations that are modally stronger than ordinary causal explanations. Other scholars, on the contrary, defend that mathematical explanations in science are not consistent with scientific explanations. For example, Steiner (1978) argues that mathematical explanations in science are subsumed under the topic of pure mathematical explanation. In this paper, I do not take sides on this matter. 
I revitalise Carl Hempel's model of scientific explanation - the deductive-nomological model of explanation (hereafter, the DN model). In this regard, I introduce mathematics and eliminate the requirement of the laws of nature in the DN model (hereafter, the DN-M model). I test this DN-M model on some well-known examples of the recent literature on mathematical scientific explanations. These examples include the bridges of Königsberg that cannot be crossed exactly once because they are (represented by) a connected graph that has an odd valence (Euler's theorem) (Pincock 2007). In addition, North American synchronized cicadas have a prime number of life-cycles (13 and 17 years) because prime periods minimise intersection (number theoretic theorem) (Baker 2005). Finally, the Hénon-Heiles Hamiltonian systems preserve almost all regular orbits of the system if sufficiently small perturbations on the value of energy are introduced (KAM theorem).

As far as I know, Molinini (2014) and Baron (2019) are the only proposals of a DN model for mathematics. The proposal of Molinini is different from my proposal. First, Molinini's proposal is a full extension of the DN model to mathematics. That is, he modifies the DN model to cover scientific explanations, mathematical scientific explanations and internal mathematical explanations. Instead, given the alleged counter-examples raised against the original DN model as a model for scientific explanation, I capitalise on Hempel's proposal for a model that covers only mathematical scientific explanations. Second, Molinini argues that the extension of the DN model to mathematics fails; I argue that my model for mathematical scientific explanations will be vindicated. The proposal of Baron is more consistent with my proposal. However, I see some problems on his constraint to distinguish between ordinary scientific explanations that employ mathematics and mathematical scientific explanations (I comment on this on section 5). ${ }^{1}$ My paper is an attempt to improve on these accounts and defend them from objections.

The proposal presented in this paper faces some background issues. First, in the literature, there is no such thing as consensual examples of mathematical scientific explanation. ${ }^{2}$ I do not advance new examples, as I am not interested in increasing unfruitful controversy. For the purposes of this paper, I assume that the examples above are examples of mathematical scientific explanation. Second, this paper is not a research on the genuineness of mathematical scientific explanations. My aim is much more modest. In this paper, it is an open question to know whether the explananda of the above mathematical scientific explanations can be explained by other ordinary scientific explanations. I propose only a model where the above examples of mathematical scientific explanations fit. Third, the proposed model, the DN-M model, is derived from a model, the DN model, that seems to be a philosophical corpse. However, I do not think that this is a significant disadvantage for the DN-M model. As I demonstrate in section 3.3, the DN-M model circumvents some relevant counter-examples to the DN model. Moreover, the DN-M model emphasises the role of mathematics in 
the explanation, and, prima facie, this role might be independent of other theoretical shortcomings of the original DN model, such as its positivist background. The motivation for the DN-M model is the following. Mathematics is a paradigm of deduction. Natural science is a paradigm of explanation. If a model for mathematical scientific explanation is not deductive, then no scientific explanatory model is deductive.

This paper is organised as follows. First, I detail my proposal of a deductivenomological model for mathematical scientific explanation. Second, I provide some clarifying points to this model. Third, I analyse an example of pure mathematical scientific explanation: the bridges of Königsberg. Fourth, I distinguish between mathematical scientific explanations that invoke laws of nature and ordinary scientific explanations that employ mathematics. Fifth, I analyse two examples of mixed mathematical scientific explanations, namely, the periodical North American cicadas and the Hénon-Heiles Hamiltonian systems. Finally, I analyse the repercussions of my model on causal explanations.

\section{The DN-M model}

Hempel (1965) proposes a deductive-nomological model for analysing the concept of scientific explanation. According to this model, the explanandum is explained if and only if it follows deductively from the explanans. The explanans comprises a set of lawlike statements $\left(L_{1}, \ldots, L_{n}\right)$ that represent the laws of nature and a set of sentences that represent the antecedent/initial conditions $\left(C_{1}, \ldots, C_{n}\right)$. There are four conditions of adequacy for an explanation as follows.

(1) The argument must be deductively valid.

(2) The explanans must contain the laws of nature required for the deduction of the explanandum.

(3) The explanans must have empirical content.

(4) The sentences of the explanans must be true.

Here is a proposal of a modified DN model for mathematical explanation. The explanandum is explained if and only if it follows deductively from the explanans. The explanans comprises a set of statements $\left(L_{1}, \ldots, L_{n}\right)$ that represent mathematical statements. This set of statements may also include general empirical laws of nature. The explanans includes a set of sentences that represent the antecedent/initial conditions $\left(C_{1}, \ldots, C_{n}\right)$. Regarding the original DN model, the DN-M model keeps the conditions of adequacy (1), (3) and (4) and modifies the condition of adequacy (2):

$\left(2_{M}\right)$ The explanans must contain a mathematical statement required for the deduction of the explanandum. 


\section{Clarifying the DN-M model}

\subsection{Scope}

A deductive model for mathematical scientific explanation goes back to Frege (1884). Mathematical scientific explanations presuppose that mathematical statements explain some empirical explanandum. To accomplish this, the explanans has mathematical and empirical components. We connect these two distinct components via mathematical applicability. That is, within the explanans, we apply the mathematical component to the empirical component. From this application, an inference will result - the explanandum. This inference is a deductive inference. Thus, the structure of mathematical scientific explanations is deductive. ${ }^{3}$

The explanantia must have some empirical content. That is, some of the terms of the explanantia must refer to empirical objects or empirical notions. At the same time, the explanantia must have a mathematical statement. However, the explanantia cannot include only mathematical statements. This restriction on the content of the explanantia implies that the DN-M model automatically excludes mathematical proofs from its analysis. That is, current mathematical proofs simply do not fit in the DN-M model. If the explanantia were able to include mathematical statements only, then all mathematical proofs would automatically become explanatory because all mathematical proofs are deductive arguments. Here, I do not argue for or against the existence of explanatory mathematical proofs.

Within the scope of the original DN model, we can distinguish two types of explanations: 1) scientific explanations exclusively based on the laws of nature; and 2) scientific explanations exclusively based on the laws of nature that employ mathematics (hereafter, SEEM). The DN-M model addresses two different types of explanations: 3) mathematical scientific explanations based on the laws of nature and mathematical statements; and 4) mathematical scientific explanations exclusively based on mathematical statements. All of these types of explanations require initial/antecedent conditions in the explanans. These conditions have empirical content. Hereafter, for the sake of simplicity, explanations of type 3) are called mixed mathematical scientific explanations (hereafter, MMSE); explanations of type 4) are called pure mathematical scientific explanations. In light of the DN-M model, there is a problem in the distinction between SEEM and MMSE. In section 5, I attempt to define a conceptual distinction to discern between these two types of explanations.

There is a problem surrounding the modal nature of the explananda of the DN-M model. Some explananda are actual physical phenomena; other explananda are physical impossibilities. For example, the explanandum on cicadas is an actual physical phenomenon, that is, "cicadas are likely to evolve 17-year periods". On the contrary, the explanandum on cats and sardines seems to be a physical impossibility, that is, "I 
cannot distribute exactly seven sardines evenly among my three cats without cutting any". In this paper, I do not address this debate. I simply refer to the explananda by the term "empirical facts".

According to Salmon (1989, p.127), "one asks a why-question only as a result of some sort of perplexity". We want to explain an observed phenomenon that strikes us as mysterious or remarkable, where explanations must provide understanding for these perplexities. Some of the contemporary literature on mathematical explanations follows this desideratum for why-questions. For example, Baker (2005, p.234) claims that the phenomenon to be explained must be considered "remarkable" and "mysterious"; Colyvan (2001, p.47) claims that an explanation "must make the phenomena being explained less mysterious". In light of the DN-M model, the explananda do not need to be mysterious or perplexing. Mystery and perplexity are psychological notions. An explanandum can be mysterious for one person but evident for other people. For example, eclipses are mysterious for tribal people but an amusement for contemporary educated people.

\subsection{Laws and mathematical statements}

There are two essential concepts that underpin the explanantia of the DN-M model: the concept of law of nature and the concept of mathematical statement. Regarding the concept of law of nature, I adopt the epistemology of Achinstein (1971). According to Achinstein, we can distinguish three different characteristics of the laws of nature:

(1) [a] fundamental theoretical idea a law introduces, which underlines the more superficial regularities which allows the basic regularity to be expressed, (...) (2) [the] factors the laws isolates (...) (3) the precise, often quantitative, manner in which it relates these factors. (Achinstein 1971, pp.85-6)

Characteristics (1) and (2) focus on the qualitative aspects of the laws of nature; characteristic (3) focuses on the quantitative aspect of the laws of nature. ${ }^{4}$ Given these characteristics, there are different ways to formulate a scientific explanation. That is, a scientific explanation depends on the characteristics of the law that the explanans emphasises. In some explanations, the qualitative aspects of the law are more central; in other explanations, the quantitative aspects of the law are more central. Thus, considering characteristics (1)-(3), I introduce a distinction between qualitative scientific explanations and quantitative scientific explanations.

Qualitative scientific explanations appeal to a basic fundamental theoretical idea associated with the law or factors that the law isolates to explain some specific aspect of the phenomenon to be explained. For example, to explain why a stick partially immersed in water appears to be bent, it is sufficient to invoke that all light rays 
are refracted when they undergo different denser optical media. This explanation appeals to a qualitative aspect of Snell's law - a basic theoretical idea of the law. In contrast, quantitative scientific explanations appeal to the relation between the different factors that the law of nature isolates, namely, the precise mathematical formula of the law of the nature. In these explanations, "what is important is how such factors are related quantitatively and what mathematical consequences follow from this relationship" (Achinstein 1971, p.87). Usually, these formulas are expressed symbolically. For example, if we want to explain the precise value of the refraction angle of a particular light beam, the value of this angle follows directly from the mathematical formula of Snell's law with mathematical calculations. That is, we must apply mathematics in the formula $\sin i / \sin r=n$, where $i$ is the angle of incidence, $r$ is the angle of refraction and $n$ is a constant.

Some scholars have recently proposed epistemic prescriptions for the mathematical statement of the explanans. For example, Molinini (2014, p.232) claims that the mathematical statement of the explanans must be a mathematical theorem; Baker (2005, p.235) suggests that it should be a mathematical theorem or a mathematical principle. There are some difficulties in proposing epistemic prescriptions for this type for mathematics.

From the point of view of the mathematical literature, mathematical statements face identity problems. Grosso modo, mathematics has definitions, axioms, postulates, theorems, lemmas, corollaries, elementary propositions, etc. However, this classification is ex cathedra. Mathematical statements are not univocally identified. Considering mathematical definitions $q$, the mathematical statement $x$ may be a theorem; regarding mathematical definitions $p$, the mathematical statement $x$ may be a corollary, a lemma or even an elementary mathematical proposition. For example, the proposition " $a+b=b+a$ " seems to be an elementary mathematical proposition. However, this proposition is part of many definitions, as it can represent the commutative property of a mathematical system (e.g., group rules in algebra). Moreover, there are many alternative mathematical formulations for the same theorem, although some of them are logically equivalent.

Mathematics is continuous with physics and other empirical sciences. Thus, in the same way as with the laws of nature, mathematical statements may be formulated in qualitative or quantitative ways. For example, classically, two different characterisations of the definition of a limit are common in current mathematical manuals. The following is a qualitative characterisation:

The function $f$ approaches the number $L$ as $x$ approaches the number $a$, but $x$ is different from $a$.

The following is a quantitative definition: 
Let $f$ be a function defined on some open interval that contains $a$, except possibly $a$ itself. Then,

$$
\begin{gathered}
\lim _{x \rightarrow a} f(x)=L \text { if and only if } \\
\forall_{\epsilon>0} \exists_{\delta>0}: \forall_{x \in \mathbf{R}}(0<|x-a|<\delta \Rightarrow 0<|x-L|<\epsilon)
\end{gathered}
$$

In the first case, the statement focuses a fundamental aspect of a limit. In the second case, a detailed definition is given, where symbolic terms are introduced. ${ }^{5}$ Let me clarify some points. First, I am not defending that the first characterisation is an alternative definition of limits. Second, although the first characterisation seems to be intelligible to the beginner and unacceptable to the professional, and conversely, the second statement seems to be correct for the professional and obscure for the beginner, both statements are objective. They do not depend on what individuals think of them. Third, both statements can be used in DN-M-type arguments; thus, both statements have explanatory power. ${ }^{6}$

Accordingly, there are qualitative and quantitative formulations of the laws of nature, and there are quantitative and qualitative formulations of mathematical statements. The problem now is to determine the possible relations between these characteristics within the explanans. It seems to me that qualitative laws of nature can only be related to qualitative mathematical statements. In section 5.2, I argue that this qualitative relation is the distinctive mark of MMSE in contrast to SEEM.

\subsection{DN-M and counter-examples to DN}

Several authors have challenged the biconditional relation that characterises the original DN model in both directions. On the one hand, some descriptions of phenomena seem to fit in the explanatory structure of the DN model, but intuitively, they are not genuine explanations. For example, the height of a flagpole (and the relevant laws of optics) explains the length of the shadow of the flagpole, for example, at noon, but through symmetry, the length of the shadow of the flagpole (and the relevant laws of optics), for instance, at noon, also explains the height of the flagpole. Both explanations are accounted for by the DN model, but intuitively, the second case is not an explanation (Bromberger 1966, pp.92-3). On the other hand, some descriptions of phenomena do not seem to fit in the explanatory structure of the DN model, but intuitively, they are genuine explanations. For example, Scriven (1962) argued that a stain on the carpet was caused by an ink bottle on the table that inadvertently was knocked from the table. This explanation seems to be perfectly reasonable, but it seems that we cannot lay down the laws of nature involved in the phenomenon (i.e., it does not fit in the DN model). ${ }^{7}$

These much-discussed cases are clearly problematic for the DN model. However, as a far I can see, they do not intersect the DN-M model. Recall that the DN-M model 
does not subsume the DN model. The first cases, at most, may apply mathematics to the laws of nature of the explanans. In section 5.2, I argue that these "applicability" cases are not (mixed) mathematical scientific explanations because they are quantitative explanations. None of the second cases requires an independent mathematical statement in the explanans to deduce the explanandum. Thus, none of these cases exemplifies mathematical scientific explanations.

The DN-M model would subsume the DN model if condition $\left(2_{M}\right)$ were replaced by $\left(2_{M}\right)^{*}$ :

$\left(2_{M}\right)^{*}$ The explanans must contain a law of nature or a mathematical statement required for the deduction of the explanandum.

With $\left(2_{M}\right)^{*}$ involved, the DN-M model would cover scientific explanations and mathematical scientific explanations (where internal mathematical explanations would continue out of the analysis). Scholars who welcome this enlarged model would need to address the alleged counter-examples to the original DN model. ${ }^{8}$ Given that I am only interested in developing a model for mathematical scientific explanation, the DN-M model is sufficient to accomplish this desideratum. Thus, I leave this enlarged model aside. ${ }^{9}$

\section{Pure mathematical scientific explanations}

In light of the DN-M model, the analysis of pure mathematical scientific explanations is unproblematic. In these cases, the explanandum is an instantiation of the mathematical statement of the explanans (plus some initial/antecedent conditions).

The configuration of the seven bridges of Königsberg is such that no one can cross all the bridges exactly once (Pincock 2007). The explanandum has empirical content: "the bridges of Königsberg cannot be crossly exactly once". The current mathematical explanation for this explanandum is a mathematical theorem: a connected graph has an Euler path if and only if it is not the case that either every vertex or every vertex but two is touched by an even number of edges. If a graph is Eulerian, then it is connected, and there is a path from an initial vertex $i$ to a final vertex $i$, where each edge is crossed exactly once. If we represent the bridges and islands in a graph, basically, the graph is non-Eulerian. That is, at least one of the vertices (islands) is touched by an odd number of edges (bridges). This graph is an abstraction of the bridge system, and it is a non-Eulerian graph. That is, there is an isomorphism between bridges and edges and between islands and vertices. Pincock claims that the fact that one of the vertices of the graph of Königsberg's bridges has an odd valence (i.e., the number of edges that touch one of the vertices is odd), which explains why it is impossible for people to cross the seven bridges of Königsberg exactly once and return to their 
starting point. Concretely, the deductive explanation fits in the DN-M structure as follows.

Explanans

(1) A connected graph has an Euler path if and only if it is not the case that either every vertex or every vertex but two is touched by an even number of edges. [Mathematical theorem]

(2) The bridges of Königsberg are a connected graph that has an odd valence. All the vertices (islands) of the bridges of Königsberg are touched by three edges (bridges). [Antecedent condition]

(3) The bridges of Königsberg do not have an Euler path [from (1) and (2)].

(4) If the bridges of Königsberg do not have an Euler path, the bridges of Königsberg cannot be crossly exactly once. [mixed mathematical/empirical statement]

\section{Explanandum}

$(\therefore)$ The bridges of Königsberg cannot be crossly exactly once.

\section{MMSE vS. SEEM}

\subsection{The problem}

Many scientific explanations are ontologically committed to mathematical entities, but these entities have no explanatory role in the explanations. These scientific explanations employ mathematics as a descriptive or calculational framework. However, in general, these scientific explanations are quite similar to the mixed mathematical scientific explanations: both invoke the laws of nature in the explanans, and both invoke mathematics. Thus, there is a problem of distinguishing between MMSE and SEEM. In this section, I shed some light on the relation between the mathematical component and the nomological component of our scientific explanations.

This is a difficult problem. Lange (2013) put forward an attempt to solve this problem. He argued that mathematical scientific explanations are non-causal explanations. This characterisation removes the causal scientific explanations that employ mathematics. However, the non-causal scientific explanations that employ mathematics remain on the table. Lange claims that "there is no criterion that sharply distinguishes the distinctively mathematical explanations from among the non-causal explanations appealing to some mathematical facts. Rather, it is a matter of degree and of context" (Lange 2013, p.507). Thus, he follows a case-by-case analysis by attempting to disentangle which component the explanans emphasises. 
I agree that there is no criterion that sharply distinguishes between MMSE and SEEM. I also agree that the difference between MMSE and SEEM is a matter of context and degree. Regarding the context, different deductive explanans may explain the same explanandum. Regarding the degree, there are clear cases of MMSE, there are clear cases of SEEM, and there are some potential borderline cases. Accordingly, I cannot agree that the criterion that distinguishes MMSE from SEEM is causation. The difference between a causal explanation and a non-causal explanation is not a difference of degree. An explanation is either causal or non-causal. There is no middle ground. Causation and gradation simply do not match. I do not think that a clear-cut distinction is the correct way to distinguish between MMSE and SEEM. The difference between MMSE and SEEM is one of degree. Therefore, a gradation distinction is the right tool to analyse gradation phenomena.

To see this problem clearly, let us detail a simple example. Suppose that someone asks this question: why will the horizontal distance travelled by a projectile be the farthest if the launched angle of the projectile is $45^{\circ}$ ? From Newton's second law, we can derive an equation of motion that is a function of the launched angle $(\theta)$ of a projectile. Here are the details:

$$
\left\{\begin{array}{l}
m \ddot{x}=0 \\
m \ddot{y}=-m g
\end{array}\right.
$$

By integrating with respect to time, we obtain

$$
\left\{\begin{array}{l}
\dot{x}=v_{0} \cos \theta \\
\dot{y}=-g t+v_{0} \sin \theta
\end{array}\right.
$$

By integrating with respect to time, again, we obtain

$$
\left\{\begin{array}{l}
x=v_{0} \cos \theta t \\
y=-g \frac{t^{2}}{2}+v_{0} \sin \theta t
\end{array}\right.
$$

By eliminating $t$ from the system of equations, we obtain

$$
\begin{aligned}
& y=\frac{-g}{2 v_{0}^{2} \cos ^{2} \theta} x^{2}+x \tan \theta \\
& \text { If } y=0, \text { then } x=\frac{v_{0}^{2}}{g} \sin (2 \theta)
\end{aligned}
$$

where $v_{0}$ is the initial velocity, $g$ is the gravitational acceleration, $\theta$ is the angle at which the projectile is launched, $x$ is the horizontal distance and $y$ is the vertical distance. The horizontal distance travelled by the projectile is at its maximum if $\sin (2 \theta)=1$, that is, if the launched angle of the projectile is $45^{\circ} .{ }^{10}$ 
This explanation has the shape of a (enthymematic) deductive argument. It employs mathematical statements. The explanans has true laws of nature and empirical content. Thus, this explanation fits in the DN-M model, and it is an MMSE. However, intuitively, the mathematical statements of the explanans do not seem to have any explanatory role in the explanation. We use the fundamental theorem of calculus to integrate the differential equations, and we use the Euclidean postulates of geometry to explain the mathematical fact of $\sin \left(90^{\circ}\right)=1$ (namely, the postulate that says that the sum of the internal angles of a triangle is two right angles). Instead, we could have applied, for example, the mathematical technique of Lagrange multipliers. From this mathematical technique, it also follows that the range of the projectile is the maximum if the vertical and horizontal initial velocities are equal, i.e., $\theta=45^{\circ} .{ }^{11}$ Accordingly, mathematics is only a calculational framework within the explanans. Intuitively, this explanation is a SEEM. The DN-M model simply does not capture this intuition. The DN-M model, as it stands, is incapable of distinguishing between MMSE and SEEM.

\subsection{Distinction QQ}

Considering the distinctions made in section 3.2, I propose that MMSE are qualitative explanations and SEEM are quantitative explanations. In the first case, the explanans invokes laws of nature and mathematical statements in a qualitative way. The mathematical component of the explanation is independent of the nomological component. In the second case, the explanans invokes laws of nature in a quantitative way. We apply mathematics directly to the mathematical formula of the respective law of nature to extract the explanandum regardless of the qualitative/quantitative nature of the mathematical statements. These explanations aim to obtain a rigorous derivation of the explanandum from the mathematical formula expression of the law of nature, where mathematics is only instrumentally used. We call this the distinction QQ.

In the projectile example, for example, we want to derive a quantitative explanandum: why is a projectile hurled farthest at a release angle of $45^{\circ}$ ? We begin by identifying the laws of nature that govern this type of motion, namely, the precise mathematical physical formulas that relate to the factors that Newton's second law isolates (force, mass and acceleration). Then, we apply mathematics to extract the explanandum from the explanans. This is a quantitative explanation, and we need to follow a precise and rigorous calculation where mathematics is an auxiliary device in the calculation process. Here, mathematics does not seem to have any explanatory role. ${ }^{12}$

The following is the process for MMSE. We formulate a deductive argument where the explanandum is the conclusion of the argument. Along this process, we invoke qualitative aspects of the laws of nature and qualitative aspects of mathematics. These qualitative aspects need not be precise and exhaustive. The qualitative 
mathematical statements and the qualitative laws of nature are premises of the explanans. Within the explanans, the mathematical statements conjoin with the laws of nature to form "new" premises. That is, the laws of nature and the mathematical statements of the explanans imply mixed nature/mathematical laws. These new mixed nature/mathematical laws are qualitative laws, and they are part of the set of the laws of the nature of the explanans. The initial conditions apply under the mixed nature/mathematical laws. It is deduced the explanandum. ${ }^{13}$

In the construction of the deductive argument, the initial conditions, the laws of nature and the mathematical statements that are relevant to explain the explanandum should be selected. The laws of nature, mathematical statements and initial conditions are classified as irrelevant to explain a particular explanandum if when added to a DN-M argument, the same particular explanandum continues to be deduced. ${ }^{14}$ Thus, irrelevance should be addressed by a comparison of the arguments. All things being equal, simpler arguments are more explanatory than other arguments (see section 6.1 below for my discussion of an example advanced by Baron).

A quantitative explanation does not require that the respective explanandum be a numerical explanandum. For instance, we can derive Galileo's formula-law from Newton's formula-laws with calculations. This derivation is a quantitative explanation, but Galileo's formula-law is not a numerical explanandum. Analogously, a qualitative explanation does not require that the respective explanandum be a non-numerical explanandum. For instance, Baker's explanation on cicadas (section 6.1) is a qualitative explanation, but the explanandum has numerical terms. It happens that the numerical term, "17", is the most relevant term of the explanandum.

SEEM are more precise and complete than mixed mathematical scientific explanations. MMSE are sketches of the scientific explanations that employ mathematics. That is, if a scientific explanation is already available for the phenomenon, we step back and attempt to disentangle the mathematical component from the nomological component. The derivation of Baker's explanation of cicadas (section 6.1) from the original scientific papers (Goles et al. 2001; Yoshimura 1997) is a good example of this procedure. If not, the qualitative explanation may be a first attempt to determine what is occurring with the phenomenon. For example, see the comments below (section 6.2) of Hénon about the role of the KAM theorem on Hénon-Heiles systems.

Two opposite objections press the distinction QQ. On the one hand, in some MMSE, intuitively, the propositions of the mathematical component may not play any explanatory role, despite the qualitative relation between the mathematical component and the law component. Thus, some MMSE may be not genuine mathematical scientific explanations and instead, may be scientific explanations that employ mathematics. On the other hand, in some SEEM, intuitively, the propositions of the mathematical component may play an explanatory role, despite the quantitative relation between the mathematical component and the law component. That is, some SEEM 
may actually be genuine mathematical scientific explanations.

First, I am not denying that MMSE and SEEM may explain the same explanandum and rest on the same laws of nature. The philosophical jargon for this phenomenon is "explanatory overdetermination". An explanation is overdetermined if two or more non-equivalent explanans-sets can explain the same explanandum. Overdetermination is not necessarily problematic. An infinite number of explanations may explain the actual state of a given physical system, as there are infinite ways to refer to the initial time (initial condition) for the system. The distinction QQ does not block explanatory overdetermination. However, the explanatory overdetermination of MMSE and SEEM does not imply that MMSE reduce to SEEM or vice-versa, as MMSE and SEEM are different types of explanations. ${ }^{15}$

Second, this paper is a defence neither of the existence of genuine mathematical scientific explanations nor of scientific explanations that employ mathematics. In the beginning of the paper, I assumed that there are mathematical scientific explanations, regardless of whether or not they are genuine, where some of them invoke laws of nature and mathematical statements (MMSE). The scientific literature is full of scientific explanations that employ mathematics (SEEM). The main aim of this paper is to provide a model - , i.e., a structure — for mathematical scientific explanations. I am not providing any model for ordinary scientific explanations that employ mathematics.

However, I admit that the distinction QQ might have a weakness on this point. The DN-M model derives from a model for scientific explanation, thus, there might be some overlap between MMSE and SEEM. However, I think that the distinction QQ might be supplemented by an additional constraint.

In MMSE, the mathematical and law components remain fixed across numerical "deformations" of the explanandum. ${ }^{16}$ That is, if we modify the numerical values of the explanandum, the mathematical and law components remain fixed, and the values of the initial conditions modify. The case of the cicada example is paradigmatic (section 6.1 below). The explanandum that "cicadas are likely to evolve 17-year periods" links to the ecological constraint (i.e., initial condition) that "cicadas are limited by biological constraints to periods from 14 to 18 years". The explanandum that "cicadas are likely to evolve 13-year periods" links to the ecological constraint (i.e., initial condition) that "cicadas are limited by biological constraints to periods from 10 to 14 years". In both explanations, the mathematical and law components remain fixed. ${ }^{17}$

In SEEM, the mathematical component changes as the numerical values of the explanandum deforms. It is not fixed. Recall that according to my view, in SEEM, the law component is a mathematical law statement. That is, the law is expressed by a function, precisely, a $\mathbf{R}^{n} \rightarrow \mathbf{R}^{m}$ function. Thus, a numerical deformation of the values of the explanandum implies deformations in the calculus of the law formula. 
The mathematical component of SEEM is fluctuant. In the projectile example above, if we "deform" the value of the horizontal distance travelled by the projectile (explanandum), then we must "deform" the angle at which the projectile is launched (initial condition). However, this numerical deformation implies that different numerical calculations apply within the law formula of the explanans. The numerical deformation changes the mathematical component.

Terminologically, it seems that two other types of explanations are possible: 1) quantitative mixed mathematical scientific explanations, i.e., qualitative mathematics is applied to the quantitative aspects of the laws of nature; and 2) qualitative scientific explanations that employ mathematics, i.e., quantitative mathematics is applied in the qualitative aspects of the laws of nature. That is, in total, it seems that there are four types of explanations. Prima facie, I do not see how it is possible to relate qualitative notions and quantitative notions. I do not address these conceptual "botanisings". From my perspective, only a closer analysis of the putative exemplification of these cases may indicate the content of the explanantia and effectively determine the type of explanation under inspection.

By putting everything together, we obtain the following necessary and sufficient conditions for MMSE. The explanandum of MMSE is explained if and only if it follows deductively from the explanans. The explanans comprises a set of qualitative statements $\left(L_{1}, \ldots, L_{n}\right)$ that represent mathematical statements and general empirical laws of nature. The explanans includes a set of sentences that represent the antecedent/initial conditions $\left(C_{1}, \ldots, C_{n}\right)$. If the explanandum is numerical, the MMSE satisfies the following counterfactual numerical requirement: if the numerical value of the explanandum had been different, then the values of the initial conditions would have been different, and the mathematical and law components would have been unchanged.

Sam Baron (2019) proposes the following constraint for extra-mathematical explanations.

A non-mathematical claim $P$ is essentially deducible from a premise set $S$ that includes at least one mathematical sentence $M$ just when there is a sound derivation of $P$ from $S$ and either there is no sound derivation of $P$ from a premise set $S^{*}$ that includes only physical sentences or all sound derivations of $P$ from premise sets $S_{1} \ldots S_{n}$ each of which includes only physical sentences are worse than the mathematical derivation. (Baron 2019, section 4)..$^{18}$

Then, he considers the following explanation for the arriving of train $T$ at station $S$ at $3: 00 \mathrm{pm}$, which departs from $S^{*}$ at $2: 00 \mathrm{pm}$, where $S^{*}$ is $10 \mathrm{~km}$ away from $S$, and $T$ is travelling at $10 \mathrm{kph}$.

Then, he considers the following explanation for the arriving of train $T$ at station $S$ at $3: 00 \mathrm{pm}$, which departs from $S^{*}$ at $2: 00 \mathrm{pm}$, where $S^{*}$ is $10 \mathrm{~km}$ away from $S$, 
and $T$ is travelling at $10 \mathrm{kph}$.

[P1] $T$ left $S^{*}$ at $2: 00 \mathrm{pm}$.

[P2] $S^{*}$ is 10 kilometers away from $S$.

[P3] $T$ is travelling at $10 \mathrm{kph}$.

[P4] For any number $m \mathrm{~m} / \mathrm{m}=1$.

[P5] If for any number $m m / m=1$, then $10 / 10=1$.

[P6] If $T$ left $S^{*}$ at $2: 00 \mathrm{pm}, S^{*}$ is 10 kilometers away from $S, T$ is travelling at $10 \mathrm{kph}$, and $10 / 10=1$, then $T$ arrives at $S$ at $3: 00 \mathrm{pm}$.

[P7] $T$ arrives at $S$ at $3: 00 \mathrm{pm}$. (Baron 2019, section 4)

He claims that this is not a genuine extra-mathematical explanation because there is an alternative derivation that includes only physical claims. However, malheuresement, this claim is not adequately justified: he does not advance any example of an alternative derivation that includes only physical claims. As he admits "that most (if not all) uses of mathematics in science can be formulated into an argument in much the same manner", then a nominalist committee must be ready to reformulate most of the physical explanations that employ mathematics.

On the contrary, in light of my distinction QQ, this is not MMSE. The premise [P4] is not a qualitative mathematical statement. Moreover, if the explanandum is deformed, then the mathematical component of the explanans must be changed. For example, to explain why train $T$ arrives at station $S$ at $4: 00 \mathrm{pm}$, if it departs from $S^{*}$ at $2: 00 \mathrm{pm}$, where $S^{*}$ is $10 \mathrm{~km}$ away from $S$, and $T$ is travelling at $5 \mathrm{kph}$, the mathematical premise [P4] must be modified, that is, "for any number $m, 2^{*} \mathrm{~m} / \mathrm{m}=$ 2".

\section{MMSE}

Let us analyse two examples of MMSE, namely, the periodical North American cicadas and the Hénon-Heiles Hamiltonian systems.

\subsection{Cicadas}

Baker (2005) argues that the 13- and 17-year life-cycles of three species of cicadas (from the genus Magicicada) from North America is a case of genuine mathematical explanation. These cicadas live underground as nymphs most of the time of their life-cycle. The adult period of the life-cycle is very brief, only several weeks (4 to 6 weeks). The mature cicadas mate, lay eggs, and their life-cycle is complete. Surprisingly, all members of a population of these species emerge synchronised. That is, they appear every thirteenth/seventeenth year. No other species of cicadas are 
synchronized. The life-cycle periods of these cicadas are prime numbers (13- and 17year life-cycles). According to biologists, these prime life-cycle periods minimises the chances of predators and hybridisation with similar subspecies. ${ }^{19}$

The structure of the explanation for an ecosystem with a 17-year life-cycle is as follows (see (Baker 2005, p.233):

Explanans

(1) Having a life cycle period which minimizes intersection with other (nearby/ lower) periods is evolutionarily advantageous. [law of nature]

(2) Prime periods minimize intersection (compared to non-prime periods). [number theoretic theorem]

From (1) and (2):

(3) Organisms with periodic life-cycles are likely to evolve periods that are prime. ['mixed' biological/mathematical law]

(4) Cicadas in ecosystem-type, E, Magicicada septendecim, are limited by biological constraints to periods from 14 to 18 years. [ecological constraint]

Explanandum:

(.) Cicadas in ecosystem-type, E, Magicicada septendecim, are likely to evolve 17year periods.

This explanation fits in the DN-M model and is MMSE. The explanans contains a law of nature (1), a mathematical statement (2) and an antecedent condition (4). This explanans explains why the cicadas in ecosystem E evolve 17-year periods. The explanation meets the four conditions of adequacy. The argument is deductively valid. The explanans has a law of nature (1) and a mathematical statement (2); these statements are required for the deduction of the explanandum. Moreover, the explanans has empirical content ((1) and (4)). Finally, the scientific statements of the explanans are supposedly true, as they belong to our corpus of best scientific theories and mathematical theorems.

This explanation does not proceed along the lines of an ordinary scientific explanation that employs mathematics. Baker's explanans invokes a biological law and a mathematical statement in a qualitative way. The explanans does not have any mathematical-biological symbolic formula; the mathematical theorem is a qualitative mathematical statement. This explanation does not apply mathematics to a putative biological law formula to derive the explanandum. The mathematical component of the explanans is independent of the laws of nature invoked in the explanans.

It may be criticised that some of the terms of premises (1) and (2) are quantitative terms. For example, "life cycle" and "prime periods" are terms that we can 
measure. Thus, this explanation is a quantitative explanation; in particular, it is one more SEEM.

The distinction QQ does not stipulate that the terms of our statements cannot be quantitative terms. For example, the second law of Newton, if formulated in the following terms, "forces cause changes in the velocity of bodies", has several quantitative terms but expresses a fundamental theoretical idea associated with the law. However, if we want to derive a quantitative explanation, the traditional mathematical formula of the law " $f=m \cdot a$ " has to be used. It is also important to emphasise that in the mathematical literature, there is no such thing as a number theoretic theorem that says "prime period minimises intersection (compared to non-prime periods)". We do not need to have a profound mathematical education to see that this theorem has a lack of rigour and clarity. For example, it is not clear what the term "intersection" means if this term is not mathematically defined in advance. The socalled "number theoretic theorem" is a qualitative creation of Baker that sums up the mathematics applied to the cicadas' explanation (in particular, it follows from two previous lemmas (Baker 2005, p.232)) to create an MMSE.

Baron illustrates the problem of irrelevance with the following argument on Baker's cicadas:

[P1*] Having a life-cycle period which minimises intersection with other (nearby / lower) periods is evolutionarily advantageous.

$[\mathrm{P} 2 *] 2+2=4$.

[P3*] If having a life-cycle period which minimises intersection with other (nearby / lower) periods is evolutionarily advantageous, and $2+2=4$ then organisms with periodic life-cycles are likely to evolve periods that are prime.

[P4*] Cicadas in ecosystem-type, $E$, are limited by biological constraints to periods from fourteen to eighteen years.

[P5*] If organisms with periodic life-cycles are likely to evolve periods that are prime and cicadas in ecosystem-type, $E$, are limited by biological constraints to periods from fourteen to eighteen years, then cicadas in ecosystem-type, $E$, are likely to evolve seventeen-year periods.

[P6*] Cicadas in ecosystem-type, $E$, are likely to evolve seventeen-year periods. Baron (2019, section 5)

Considering what I said above on irrelevance, if we delete [P2*] and the "and $2+2=4$ " of $\left[\mathrm{P} 3^{*}\right]$, we obtain a simpler argument that implies the same conclusion [P6*]. All things being equal, [P2*] and "and $2+2=4$ " in [P3*] are irrelevant mathematical statements to explain [P6*]. Thus, this simpler argument is more explanatory than the above argument proposed by Baron. ${ }^{20}$ 


\subsection{Hénon-Heiles systems}

The Hénon-Heiles systems are Hamiltonian systems for the study of stellar dynamics. Before Hénon and Heiles (1964), only two isolating integrals of the equations of motion were known for the motion of stars in axisymmetric galaxies. Empirical evidence suggested that a third isolating integral of motion existed. However, no one had found the analytical general form for this third integral. Based on numerical calculations, Hénon and Heiles established the condition (the value of a tuneable parameter - total energy) for the existence of this third integral of motion. The data obtained from numerical calculations were plotted in two-dimensional graphics - phase spaces (also known as phase portraits) — of position vs. momentum. High-energy Hénon-Heiles systems exhibit chaotic and unpredictable motion, and low-energy Hénon-Heiles systems exhibit regular and predictable motion.

Lyon and Colyvan (2007) defend the alleged explanatory power of phase spaces under the Hénon and Heiles model (1964) for the study of stellar dynamics. After providing some figures of phase portraits, the main conclusions of Lyon and Colyvan are as follows:

These are explanations that can be obtained from an analysis of the system using its phase space, but which cannot be obtained otherwise. (...) Instead of studying the phase space, we could look at the various paths in the $q y-q x$ plane that the star can take for different energy levels. But such an analysis would be extremely tedious (to say the least) and this just does not give us the same kind of understanding as the phase-space analysis does. The explanatory power is in the structure of the phase space and the Poincaré map. (Lyon and Colyvan 2007, p.238, p.240)

It seems that here, there are some misunderstandings of what is occurring. The explanandum is the following: high-energy Hénon-Heiles systems exhibit chaotic and unpredictable motion, and low-energy Hénon-Heiles systems exhibit regular and predictable motion. In the explanans, there is the Hamiltonian of the system (a law of nature) that depends on the analytical form of the potential found by Hénon-Heiles (a third-order potential). The equations of the motion of the system follow from the Hamiltonian of the system. The equations of motion depend on the energy of the system. That is, considering the value of total energy, star orbits may exhibit chaotic and unpredictable motion or regular and predictable motion. How do we know this? The values obtained by numerical calculations show that the orbit of a test star would have these two types of motions.

The data collected may be represented in phase spaces (with the help of a Poincaré map) through a phase portrait. In this case, the phase space is used for plotting the dynamical states of position vs. momentum when the test star revolves around an axisymmetric axis. Phase space portraits display the content of the equations of the 
motion of a system for different parameters. They are an artefact for the analysis to represent the data obtained from numerical computations. Other representations are still possible (such as "the $q y-q x$ plane", i.e., the position plane $(x, y)$ ). Lyon and Colyvan say that this "analysis would be extremely tedious". However, the analysis made by Hénon and Heiles was also extremely tedious at the time. ${ }^{21}$ It simply seems that explanatory power is independent of psychological states such as tedium. Moreover, there are other methods to determine chaotic motions, namely, the power spectrum method, Lyapunov exponents, Kolmogorov-Sinai entropy, etc. (Argyris et al. 2015, p.28; Frigg 2004, p.415). Thus, the analysis of phase spaces is instrumental - a quick and easy representation of motion.

Let us consider the motion of an undamped simple pendulum. The phase portrait of the motion of this pendulum is an ellipse. Why does this pendulum have a regular motion? Clearly, the answer cannot be that the phase portrait is an ellipse. The explanation for the observed regularity follows from the equations of the motion of the pendulum, i.e., the laws of nature that govern the behaviour of this type of object and the initial conditions of the system. Now, let us consider the motion of an autonomous double-pendulum. Why does this pendulum have a chaotic motion? Clearly, the answer cannot be that the phase portrait is not "regular". Again, the explanation for the chaotic motion follows from the equations of motion, i.e., the laws of nature that govern the physics of this type of object and the initial conditions of the system. The power of visual-geometrical analysis simply evaporates for phase portraits above three dimensions.

The philosophical discussion around this type of system goes back to Malament's (1982) review of Field's book Science Without Numbers (Field 1980). Malament claims that Field's strategy cannot be extended to classic Hamiltonian mechanics because Hamiltonian mechanics is a phase space theory, and phase space theories quantify over possible dynamical states (i.e., abstract objects). Following this line of thought, it may be challenged that, in the end, phase spaces theories, such as the Hamiltonian theory, quantify over possible dynamical states (i.e., abstract objects), and phase spaces theories are thus essential to our explanation. This objection is correct, but the point raised before is independent from Malament's claim. I distinguish between portraits of phase spaces and theories and simply argue that the analysis of data in terms of portraits of phase spaces does not have an explanatory power of the dynamics of a system. Portraits of phase spaces are simply a representation of the dynamics of a physical system.

In light of the Hénon-Heiles systems, Molinini defends that the extension of the DN model to mathematics fails because "it cannot deal with mathematical operations or procedures that do not come under the form of statements but which are regarded as playing an explanatory role". That is, "the mathematical component of the explanation does not come in the form of a theorem" (Molinini 2014, p.234). This claim 
is incorrect for two different reasons.

First, some mathematical operations can be used in the explanans, but it does not automatically follow that the use of mathematics has an explanatory role in the process of explanation. As I argued before, typically, scientific explanations use mathematics as a calculational framework. Second, to study the Hénon-Heiles systems, we need a robust mathematical statement - the KAM theorem (named after Kolmogorov, Arnold and Moser). Moreover, this theorem is part of the explanans of a mixed mathematical scientific explanation to explain why a system preserves almost all its regular orbits if we introduce sufficiently small perturbations to the value of the energy of the system. Molinini, Lyon and Colyvan simply neglected this theorem in their philosophical analysis.

For Hénon and Heiles the explanandum was simply astounding.

In some cases the star orbits were quite regular, in the usual way, but in other cases they behaved wildly, jumping here and there in an apparently random fashion. These results were hard to believe; the people who saw them, including us, were skeptical and wondered about a possible bug in the program. (Hénon 1988)

They started to believe that something was wrong in their calculations and that the third integral of motion did not exist. However, the qualitative aspect of the KAM theorem was central to the explanandum.

By a fortunate coincidence V. Arnold and J. Moser, working independently, had at the same time obtained their proofs of what was to become famous as the KAM theorem. In December 1962 I attended a gathering of astronomers at Yale. Moser was present and gave an illuminating presentation of the latest mathematical results and their consequences for the dynamics of nonintegrable systems. Suddenly everything fell into place: qualitatively at least, the mathematical theory completely explained the strange mixture of order and chaos found in our numerical results. (Hénon 1988, italics mine)

Basically, the KAM theorem concerns Hamilton systems and perturbation theory. An integrable system (which is not degenerate and is sufficiently differentiable) has the property that most of the quasi-periodic orbits are preserved for sufficiently small perturbations. $^{22}$

Let us attempt to apply the DN-M model to the Hénon-Heiles systems. In the explanans, there is the Hamiltonian of the system (a law of nature) that depends on the analytical form of the potential found by Hénon and Heiles (a third-order potential). The equations of the motion of the system follow from the Hamiltonian of the system. The equations of motion depend on the energy of the system. A qualitative interpretation of these equations is "the number of the orbits of a Hamiltonian HénonHeiles system is a function of the energy of the system". There are also some initial 
conditions (e.g., the value of total energy) and the KAM theorem. The structure of the explanation is the following:

Explanans:

(1) The number of the orbits of a Hamiltonian system is a function of the energy of the system. [physical law]

(2) If sufficiently small perturbations on the value of the energy of a Hamiltonian system are introduced, almost all regular orbits of the system are preserved. [KAM theorem]

(3) If sufficiently small perturbations on the value of the energy of a Hamiltonian system are introduced, the variation of the number of regular orbits is very small. ["mixed" physical/mathematical law, from (1) and (2)]

(4) The values of energy $a_{i}$ are sufficiently small perturbations on the value of the energy of the test star $S$ of the Hamiltonian Hénon-Heiles system $H$. [initial condition, for $i=1$ to $n$ ]

\section{Explanandum:}

$(\therefore)$ The variation of the number of regular orbits of test star S of the Hamiltonian Hénon-Heiles system $H$ is very small. (That is, system $H$ preserves almost all regular orbits of test star $S) .^{23}$

This is a qualitative explanation. In particular, this is MMSE. The law of nature and the initial conditions are insufficient to explain why the number of regular orbits is almost unchanged when small perturbations on the value of energy are introduced. This structure obeys the conditions of adequacy. The argument is deductively valid. The explanans contains a law of nature (1) and a mathematical statement (KAM theorem). The explanans has empirical content (4). The sentences of the explanans are true, the physical law is true, the initial conditions are observational facts (numerical inputs for test star $S$ ) and there is a proof of the KAM theorem.

\section{Mathematical scientific explanations and causation}

A last knot remains to be untied. According to the original DN model of scientific explanation, the concept of causation is subsumed under the concept of explanation. Thus, the DN model addresses non-causal and causal explanations. On the one hand, general laws or theoretical principles can explain particular laws. For example, the DN model can account for why Newton's laws of motion and gravitation (some approximations must be introduced) can explain Galileo's law. Typically, these types of explanations are non-causal explanations. On the other hand, the DN model can 
also account for why the event-token $c$ caused the event-token $e$. The explanans has a relevant covering causal law, where event-token $c$ and event-token $e$ are instances of the law. The explanans has initial conditions for event-token $c$. The explanandum - event-token $e$ - deductively follows from the explanans. For example, to explain why the rise of temperature increases the length of the rails of Gare Saint-Lazare, the explanans must have a covering law (a relation between the temperature and the length of iron bars) and some initial conditions. Typically, these types of explanations are causal explanations (Hempel 1965, p.300). Given that the DN-M model is quite similar to the DN model, the DN-M model is also consistent with non-causal and causal explanations.

To the best of my knowledge, the contemporary literature thoroughly rejects the putative existence of causal mathematical scientific explanations (e.g., Baker (2005, p.234) and Lange (2013, p.487)). The following is the argument. Grosso modo, a causal theory of explanation argues that to explain it is to see how an event is produced by a causal mechanism such as a causal process, a causal interaction or a causal law (Salmon 1984, p.132); to explain is to provide "some information about its causal history" (Lewis 1986, p.217). However, according to mathematical Platonism, mathematical statements refer to existent mathematical abstract objects. Mathematical abstract objects are not located in space-time, i.e., mathematical abstract objects are causally inert; thus, they cannot causally be related to anything else. There are no causal links between objects not located in space-time and events in space-time. Thus, in mathematical scientific explanations, if the explanans is a mathematical statement and the explanandum is a space-time event, it is not evident how to establish an explanatory connection between the explanans and the explanandum. Thus, causal theories of mathematical scientific explanations are problematic. Prima facie, either mathematical platonic objects must come down to earth or space-time events must ascend to the heavens.

However, non-causal accounts of mathematical scientific explanations are also problematic. For example, Lange (2013, p.488) argues that the mathematical fact "that twenty-three cannot be divided evenly by three, explains why Mother failed (...) to distribute her strawberries evenly among her children". This structure of explanation is not a deductive argument but a statement (say, $q$ because $p$ ): the explanans is a mathematical fact (and nothing else is required for the explanans), and the explanandum is a space-time event. Lange indicates that mathematical scientific explanations are non-causal explanations because they are explanations that describe the framework of the causal relations. Mathematical explanations are similar to spatiotemporal symmetry principles. The difficulty is Lange's alleged equivalence between description and explanation. I can say that the raven in my backyard is black. However, this description does not explain why the raven is black.

The DN-M model is consistent with the non-causal accounts of mathematical sci- 
entific explanations. Pure mathematical scientific explanations are non-causal explanations. However, some MMSE are also non-causal explanations, as the insertion of a mathematical statement in the explanans is consistent with the non-causal laws on the structure of an explanation. For example, the structure of the explanation of Hénon-Heiles systems, outlined above, is a non-causal explanation. Only theoretical statements constitute the structure of the explanation. Computational simulations establish the explanandum. The explanandum is not a space-time event. It is a theoretical consequence of calculations. The laws of nature and a mathematical statement (KAM theorem) constitute the explanans. Thus, nothing in the explanans causally explains the explanandum. As I see it, this example is similar to the derivation of Galileo's law from Newton's laws of motion and gravitation. General laws and a mathematical theorem allow deducing the general form of the orbit of stars.

The DN-M model is also consistent with the causal explanations of mathematical scientific explanations. Mathematical statements and general causal laws may constitute the explanans. That is, the explanans may have causal and non-causal statements. The non-causal statements are mathematical statements (and some empirical non-causal laws, if needed). The causal statements are empirical causal laws. Some explanations intuitively seem to be mathematical despite being causal explanations. These cases motivate a model for mathematical explanation with room for causal mathematical scientific explanations. Explanation aims towards understanding. Understanding aims to obtain more knowledge around the explanandum. Knowledge is independent of causation. We can obtain more knowledge by knowing the causes of the explanandum; we can obtain more knowledge by knowing how to deduce an explanandum from general laws. It seems to me that to put causation before explanation is to reverse the proper order. If a mathematical scientific explanation seems to be a causal explanation, then so be it.

For example, Lange (2013, p.499) argues that Baker's own explanation of why cicada life-cycle periods are prime does not qualify as a distinctively mathematical explanation because it is a causal explanation. Baker's structure of explanation uses a "bit of mathematics", but its explanatory power is derived from the selection laws, namely, the natural history of the cicadas of North America. According to him, this explanation is a causal explanation similar to other ordinary causal explanations.

I agree that Baker's own explanation is a causal explanation; however, I disagree that it is not a mathematical scientific explanation. Considering the DN-M model, I have argued that Baker's structure of explanation is MMSE. This explanation is not an ordinary scientific explanation that employs "a bit of mathematics", where the mathematics is a calculation device in the explanatory process. The "bit of mathematics" is the number theoretic theorem. This explanation is not a quantitative explanation. Rather, this explanation is a qualitative explanation. If we exclude this theorem from the explanans, then we cannot deduce the explanandum from the explanans. The 
number theoretic theorem has an explanatory role in the process of explanation.

\section{Conclusion}

In this paper, I argue for a deductive-nomological model to square mathematical scientific explanations. The original DN model is insufficient to align these explanations. I argue that these explanations can be addressed if independent mathematical statements are inserted in the explanans structure of the original DN model.

\section{References}

Achinstein, P. 1971. Law and Explanation. Oxford: Oxford University Press.

Argyris, J. H.; Faust, G.; Haase, M.; Friedrich, R. 2015. An Exploration of Dynamical Systems and Chaos. Berlin, Heidelberg: Springer Berlin Heidelberg.

Baker, A. 2005. Are there Genuine Mathematical Explanations of Physical Phenomena? Mind 114(454): 223-38.

Baker, A. 2012. Science-Driven Mathematical Explanation. Mind 121(482): 243-67.

Baron, S. 2019. Mathematical Explanation by Law. The British Journal for the Philosophy of Science 70(3): 683-717.

Bromberger, S. 1966. Why-Questions. In: R. Colodny (ed.) Mind and Cosmos: Essays in Contemporary Science and Philosophy, pp.86-111. Pittsburgh: University of Pittsburgh Press.

Colyvan, M. 2001. The Indispensability of Mathematics. New York: Oxford University Press.

Daly, C.; Langford, S. 2009. Mathematical Explanation and Indispensability Arguments. The Philosophical Quarterly 59(237): 641-58.

Dedò, M. 2017. Q for Qualitative Mathematics. Lettera Matematica 5(2): 167-71.

Díez, J. 2014. Scientific w-Explanation as Ampliative, Specialized Embedding: A Neo-Hempelian Account. Erkenntnis 79(8): 1413-43.

Field, H. 1980. Science Without Numbers. Princeton, NJ: Princeton University Press.

Frege, G. 1884. Der Grundlagen die Arithmetik. Breslau: W. Koebner.

Frigg, R. 2004. In What Sense Is the Kolmogorov-Sinai Entropy a Measure for Chaotic Behaviour? Bridging the Gap between Dynamical Systems Theory and Communication Theory. The British Journal for the Philosophy of Science 55(3): 411-34.

Goles, E.; Schulz, O.; Markus, M. 2001. Prime number selection of cycles in a predator-prey model. Complexity 6(4): 33-8.

Hempel, C. 1964. On the Nature of Mathematical Truth. In: P. Benacerraf; H. Putnam (eds.) Philosophy of Mathematics, pp.377-93. Cambridge, Mass.: Cambridge University Press.

Hempel, C. 1965. Aspects of Scientific Explanation and Other Essays in the Philosophy of Science. New York: The Free Press.

Hénon, M. 1988. Citation Classic - The Applicability of the 3rd Integral of Motion - Some Numerical Experiments. Current Contents/Phys Chem Earth 4: 18.

Hénon, M.; Heiles, C. 1964. The Applicability of the Third Integral of Motion: Some Numerical Experiments. The Astronomical Journal 69(1): 73-9. 
Jackson, F.; Pettit, P. 1990. Program Explanation: A General Perspective. Analysis 50(2): 10717.

Lange, M. 2013. What Makes a Scientific Explanation Distinctively Mathematical? British Journal for the Philosophy of Science 64(3): 485-511.

Lewis, D. 1986. Philosophical Papers: Volume II. New York: Oxford University Press.

Lyon, A. 2012. Mathematical Explanations of Empirical Facts, And Mathematical Realism. Australasian Journal of Philosophy 90(3): 559-78.

Lyon, A.; Colyvan, M. 2007. The Explanatory Power of Phase Spaces. Philosophia Mathematica 16(2): 227-43.

Malament, D. 1982. Review of: Science Without Numbers: A Defense of Nominalism. by Hartry Field. The Journal of Philosophy 79(9): 523-34.

Molinini, D. 2014. Deductive Nomological Model and Mathematics: Making Dissatisfaction more Satisfactory. THEORIA. An International Journal for Theory, History and Foundations of Science 29(2): 223-41.

Pincock, C. 2007. A Role for Mathematics in the Physical Sciences. Noûs 41(2): 253-75.

Poincaré, H. 1921. Foundations of Science. New York: The Science Press.

Salmon, W. 1984. Scientific Explanation and the Causal Structure of the World. Princeton: Princeton University Press.

Salmon, W. 1989. Four Decades of Scientific Explanation. Pittsburgh: University of Pittsburgh Press.

Scriven, M. 1962. Explanations, predictions, and laws. In: H. Feigl; G. Maxwell (eds.) Scientific Explanation, Space, and Time (Minnesota Studies in the Philosophy of Science) (Vol. 3), pp.170-230. Minneapolis: University of Minnesota Press.

Steiner, M. 1978. Mathematics, Explanation, and Scientific Knowledge. Noûs 12(1): 17-28.

Steiner, M. 2002. The Applicability of Mathematics as a Philosophical Problem. Cambridge, Mass.: Harvard University Press.

Yoshimura, J. 1997. The evolutionary origins of periodical cicadas during ice ages. American Naturalist 149(1): 112-24.

\section{Notes}

${ }^{1}$ I received knowledge of Sam Baron's (2019) proposal when I was finishing this paper. For lack of space and time, I cannot address his proposal in detail.

${ }^{2}$ For example, Chris Daly and Simon Langford (2009, p.656) argue that in the cicada case, the explanation is based "on the physical phenomenon of duration rather than on a mathematical theory".

${ }^{3}$ Steiner (2002, pp.15-9) shows how this deduction can be performed in light of Frege's ideas.

${ }^{4}$ In what follows, I focus on cases (1) and (3) and leave aside (2). For a development of these characteristics, see Achinstein (1971, Chapters 1, 5). Hempel (1965, pp.338-9) also emphasises that law statements can take many forms such as simple universal conditionals, universal and existential generalisations and "mathematical relationships between different quantitative variables". 
${ }^{5}$ See Dedò (2017) for more examples on topology. See Poincaré (1921, pp.430-47) for examples on mathematical definitions in mathematical teaching.

${ }^{6}$ I am grateful to an anonymous reader for emphasising this point.

${ }^{7}$ See Hempel (1965, p.360, p.423) for a reply to Scriven (1962).

${ }^{8}$ Baker seems to welcome this enlarged model, as he suggests the following modification of the original DN model to square the mathematical scientific explanations: "a broadening of the category of laws of nature to include mathematical principles theorems and principles" (Baker 2005, p.235).

${ }^{9}$ Due to space constraints, I cannot address in this paper "all" relevant past objections to the DN model. See José Díez (2014) for a neo-Hempelian account of scientific explanation, where most of these relevant objections are addressed.

${ }^{10}$ In this example, I have followed Achinstein (1971, p.88).

${ }^{11}$ Let $f\left(v_{0 x}, v_{0 y}\right)=\frac{2 v_{0 x} v_{0 y}}{g}, h\left(v_{0 x}, v_{0 y}\right)=\frac{m\left(v_{0 x}^{2} v_{0 y}^{2}\right)}{2}=E$ where $f$ is the range of the projectile, and $E$ is the fixed kinetic energy of the projectile. From

$$
\begin{gathered}
\nabla f\left(v_{0 x}, v_{0 y}\right)=\lambda \nabla h\left(v_{0 x}, v_{0 y}\right) \\
\frac{m\left(v_{0 x}^{2} v_{0 y}^{2}\right)}{2}=E
\end{gathered}
$$

it follows that $f$ is the maximum if $v_{0 x}=v_{0 y}$.

${ }^{12}$ Hempel defended this point regarding the application of mathematics to Boyle's law: "the function of the mathematics here applied is not predictive at all (...) it renders explicit certain assumptions or assertions which are included in the content of the premises of the argument [the laws of nature]" (Hempel 1964, p.390).

${ }^{13}$ The mixed nature/mathematical laws by themselves conjoined with the initial conditions are sufficient to explain the explanandum. This is a minimal explanation. However, this explanation does not cause problems for my model. A minimal explanation is not MMSE, as it is an explanation without mathematical statements in the explanans. It does not fit in the DN-M model, and is an ordinary qualitative scientific explanation. See Hempel (1965, pp.346-7) for minimal explanations of ordinary scientific explanations.

${ }^{14}$ This solution to the irrelevance is adapted from a reply of Hempel (1965, pp.420-1) to an alleged requirement of total evidence of the explanans raised by Scriven (1962, pp.229-30).

${ }^{15}$ This type of overdetermination has repercussions on the explanatory indispensability arguments in mathematics. Briefly, if two different explanans explain the same explanandum - a MMSE and a SEEM - then mathematics does not play an indispensable explanatory role to derive the explanandum, as we can explain the explanandum without a component of explanatory mathematics.

${ }^{16}$ The essential idea of an explanandum must remain unchanged across the explanandum deformations.

${ }^{17}$ The same goes for the Hénon-Heiles systems (section 6.2 below): "the number of regular orbits" (explanandum) links to "the value of energy" (initial condition).

${ }^{18}$ Baron adds some other constraints (namely, to avoid irrelevant premises). However, for the purpose of my discussion, the analysis of this constraint is sufficient to make my point.

${ }^{19}$ See Baker (2005) for a detailed analysis of this case.

${ }^{20}$ For the sake of argument, I ignore the fact that " $2+2$ " is not a qualitative mathematical statement. 
21 "There were no plotting devices available at that time, and with the help of my young wife we spent some evenings plotting hundreds of points by hand on large sheets of graph paper". (Hénon 1988)

${ }^{22}$ The details of the KAM theorem are beyond the scope of this paper.

${ }^{23}$ Accordingly, if the values of energy $a_{i}$ are "lower", then "low-energy Hénon-Heiles systems exhibit regular and predictable motion". If the values of energy $a_{i}$ are "higher", then "high-energy Hénon-Heiles systems exhibit chaotic and unpredictable motion".

\section{Acknowledgments}

I am very grateful to Carl Hoefer, José Díez and two anonymous reviewers of this journal whose detailed comments have substantially improved previous versions of this paper. I am grateful to Mário Bessa for a helpful chat on Hénon-Heiles systems. I am grateful to LOGOS, University of Barcelona, who invited me to present a version of this paper and provided a friendly atmosphere to my sabbatical visit.

This work was supported by grant SFRH/BSAB/128040/2016, Fundação para a Ciência e a Tecnologia, Programa Operacional Capital Humano. 